OPEN ACCESS

Edited by: Sarvajeet Singh Gill, Maharshi Dayanand University, India

Reviewed by: Maoteng Li,

Huazhong University of Science and Technology, China

Santosh Tiwari,

Maharshi Dayanand University, India

${ }^{*}$ Correspondence:

$X i-l i n \mathrm{Hou}$

hxl@njau.edu.cn

Specialty section:

This article was submitted to Crop Science and Horticulture, a section of the journal

Frontiers in Plant Science

Received: 27 February 2016 Accepted: 04 July 2016

Published: 26 July 2016

Citation:

Zheng J-s, Sun C-z, Zhang S-n, Hou X-I and Bonnema G (2016) Cytogenetic Diversity of Simple Sequences Repeats in Morphotypes of Brassica rapa ssp. chinensis. Front. Plant Sci. 7:1049. doi: 10.3389/fpls.2016.01049

\section{Cytogenetic Diversity of Simple Sequences Repeats in Morphotypes of Brassica rapa ssp. chinensis}

\author{
Jin-shuang Zheng ${ }^{1,2}$, Cheng-zhen Sun ${ }^{1,2}$, Shu-ning Zhang ${ }^{1}, \mathrm{Xi}_{\text {-lin } \mathrm{Hou}^{1 *} \text { and }}$ \\ Guusje Bonnema ${ }^{3}$
}

1 State Key Laboratory of Crop Genetics and Germplasm Enhancement, Key Laboratory of Biology and Germplasm Enhancement of Horticultural Crops in East China, Ministry of Horticulture, Nanjing Agricultural University, Nanjing, China, ${ }^{2}$ Hebei Normal University of Science and Technology, Qinhuangdao, China, ${ }^{3}$ Wageningen UR Plant Breeding, Wageningen University and Research Centre, Wageningen, Netherlands

A significant fraction of the nuclear DNA of all eukaryotes is comprised of simple sequence repeats (SSRs). Although these sequences are widely used for studying genetic variation, linkage mapping and evolution, little attention had been paid to the chromosomal distribution and cytogenetic diversity of these sequences. In this paper, we report the distribution characterization of mono-, di-, and tri-nucleotide SSRs in Brassica rapa ssp. chinensis. Fluorescence in situ hybridization was used to characterize the cytogenetic diversity of SSRs among morphotypes of $B$. rapa ssp. chinensis. The proportion of different SSR motifs varied among morphotypes of B. rapa ssp. chinensis, with tri-nucleotide SSRs being more prevalent in the genome of $B$. rapa ssp. chinensis. We determined the chromosomal locations of mono-, di-, and tri-nucleotide repeat loci. The results showed that the chromosomal distribution of SSRs in the different morphotypes is non-random and motif-dependent, and allowed us to characterize the relative variability in terms of SSR numbers and similar chromosomal distributions in centromeric/peri-centromeric heterochromatin. The differences between SSR repeats with respect to abundance and distribution indicate that SSRs are a driving force in the genomic evolution of $B$. rapa species. Our results provide a comprehensive view of the SSR sequence distribution and evolution for comparison among morphotypes $B$. rapa ssp. chinensis.

Keywords: Brassica rapa, simple sequence repeats, fluorescence in situ hybridization, cytogenetic diversity, heterochromatin

\section{INTRODUCTION}

Simple sequence repeats (SSRs), also known as microsatellites, are composed of 1-6 nucleotide motifs that are repeated in tandem and are widely and non-randomly distributed in 100-1000s of copies in the genomes of both monocots and dicots (Tautz and Renz, 1984; Toth et al., 2000; Mortimer et al., 2005; Lawson and Zhang, 2006; Hong et al., 2007). Microsatellites are found predominantly in heterochromatin regions, such as centromeric, peri-centromeric, and sub-distal regions of eukaryotic chromosomes (Yang et al., 2005; Li et al., 2010), and sex chromosomes in animals (Beckmann and Weber, 1992; Cuadrado and Jouve, 2011), usually associated with 
the constitutive heterochromatin (Lohe et al., 1993; Pedersen et al., 1996). Variously non-random, taxon-specific patterns of SSR occurrence call for functional interpretations. Morgante et al. (2002) hypothesized that the relative frequency of microsatellites is higher in the single- or low-copy regions of the genome than in the repetitive regions. Mortimer et al. (2005) showed that SSRs are associated with and around transcribed sequences in Arabidopsis. Li et al. (2004) reviewed that SSRs in different positions in a gene can play important roles in regulating its expression and determining the function of its products. The accumulated evidence indicates that SSRs play an important role in chromatin organization, regulation of gene activity (Nagaki et al., 2004), recombination, DNA replication, the cell cycle, the mismatch DNA repair system (Li et al., 2002), and protein coding regions (Sonah et al., 2011).

The fluorescence in situ hybridization (FISH) technique has been used to localize one or more SSR loci on chromosomes. FISH has become a strategy for chromosome diagnosis and for investigating plant genome organization (Cuadrado and Schwarzacher, 1998; Cuadrado and Jouve, 2002). SSRs change rapidly during evolution, and thus display polymorphism at homologous sites between closely related species. Begum et al. (2009) showed that the rapid evolution of repetitive DNA sequences has resulted in species-specific repeat variants and the generation of novel repeat families. This characteristic has made SSRs useful as markers in comparative diversity analysis (Zhebentyayeva et al., 2003; Yu et al., 2010; Fang et al., 2013; Zhu et al., 2013) and genetics research (Nanda et al., 1991). SSRs are highly abundant within genomes, they can be widely dispersed or be confined to certain chromosomal regions, and they display a high degree of length polymorphism (Katti et al., 2001; Lawson and Zhang, 2006). Carmona et al. (2013) defined cytogenetic diversity in terms of the differences in abundance and distribution of microsatellites, and also found some specific and motif-dependent hybridization patterns. The repeats AG, AAG, ACT, and ATC presented different in situ hybridization patterns that provided cytogenetic landmarks for chromosome identification in barley, Hordeum vulgare ssp. vulgare (Carmona et al., 2013). Altogether, such variation could be used to determine evolutionary relationships between related species.

Brassica rapa belongs to the A genome species group in the Brassicaceae with $2 n=20$ chromosomes (Nagaharu, 1935), which had a monophyletic origin (Lysak et al., 2005; Cheng et al., 2013). B. rapa comprises several sub-species, such as non-heading Chinese cabbage (B. rapa ssp. chinensis), Chinese cabbage (B. rapa ssp. pekinensis; Koo et al., 2004), and turnip (B. rapa L. ssp. Rapifera; Snowdon, 2007). B. rapa ssp. chinensis is one of the most important leafy vegetable forms in $B$. rapa, and consists of five morphotypes (Pak-choi, Wu ta cai, Cai xin, Fen nie cai, and Tai cai; Viehoever et al., 1920¹ Gladis and Hammer, 1992; Zheng et al., 2015). The phylogenetic relationships between some B. rapa ssp. chinensis morphotypes have been determined from morphological, ecological, and molecular data (Yu et al., 2010). In this paper, systematic research was performed to investigate the cytogenetic diversity between intra-specific forms of $B$. rapa.

${ }^{1}$ http://www.plantnames.unimelb.edu.au/Sorting/Brassica_rapa.html\#praecox
The objective of the work presented here is to characterize the cytogenetic diversity of SSRs between morphotypes of $B$. rapa ssp. chinensis that represent a broad range of cytogenetic diversity. The available genome sequence of Chinese cabbage is an important and fundamental resource for understanding this species ${ }^{2}$ (Wang et al., 2011), but the vast majority of heterochromatic regions remain essentially uncharacterized. The current estimates of SSR frequencies in many organisms differs from reality after comparisons with sequence databases (Hong et al., 2007; Cavagnaro et al., 2010; Gao et al., 2011). The distribution of SSRs in databases has been reported for the Chinese cabbage genome (Hong et al., 2007). In view of their ubiquity and functional importance, detailed information will be necessary to explore the comparative cytogenetics of SSRs in B. rapa.

Simple sequence repeats appear to be more abundant in noncoding regions than in coding regions of plant genomes (Hong et al., 2007; Cavagnaro et al., 2010). Tri-nucleotide repeats, the most abundant SSR types in many species (Gao et al., 2003; Shi et al., 2013), were found to be the most frequent in protein coding regions (Sonah et al., 2011). In addition to tri-nucleotide repeats, mono- and di-nucleotide repeats were also predominant in the B. rapa genome (Hong et al., 2007; Gao et al., 2011). In this study, we selected mono-, di-, and tri-nucleotide repeats for physical mapping on the chromosomes of $B$. rapa. FISH was performed with mono-, di-, and tri-nucleotides to detect the distributional profile of SSRs and to enhance our understanding of genome organization. The distributional characterization of SSRs revealed a range of cytogenetic diversity that could relate to genome organization, function, and evolutionary trends. We demonstrated that: (1) not all of the SSR-based probes produced FISH signals on all B. rapa ssp. chinensis chromosomes; (2) some SSR signal intensity did not show a relationship to the abundance in the genome database; (3) the distributional patterns of SSR signals depended on the SSR motif used and the species analyzed; and (4) differences in SSR abundance and density were shown within and between genomes.

\section{MATERIALS AND METHODS}

\section{Plant Materials}

Five morphotypes of $B$. rapa ssp. chinensis: Pak-choi (cv. NHCC002), Wu ta cai (cv. NHCC006), Cai xin (cv. NHCC008), Fen nie cai (cv. NHCC010), Tai cai (cv. NHCC015) were stored and cultivated at the Key Laboratory of Biology and Germplasm Enhancement of Horticultural Crops in East China, Ministry of Horticulture, China. All of these morphotypes have distinct phenotypic characteristics in terms of leaf shape and size, number of leaves, number of outgrowing axially buds, and flowering time (Figures 1a-e).

\section{Chromosome Preparation}

Mitotic metaphase chromosome preparation from root tips followed the procedure described by Zheng et al. (2014).

\footnotetext{
${ }^{2}$ http://brassica.bbsrc.ac.uk/brassica_genome_sequencing_concept.htm
} 


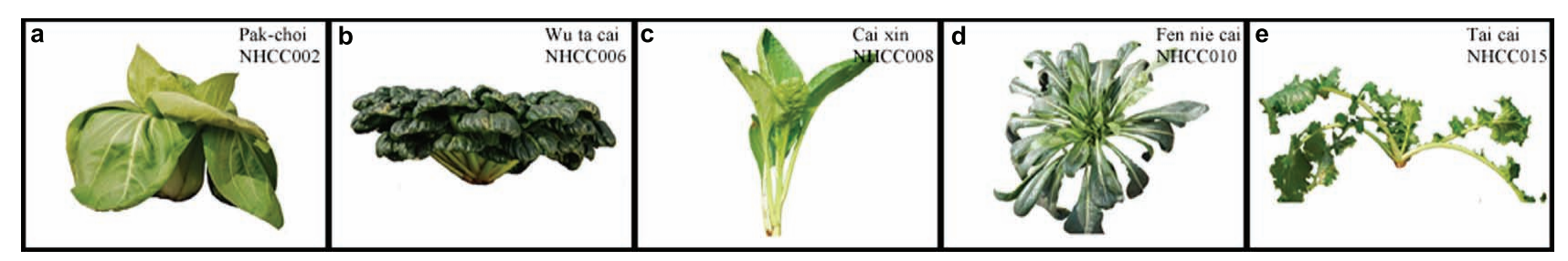

FIGURE 1 | The five morphotypes of Brassica rapa ssp. chinensis. (a) Pak-choi; (b) Wu ta cai; (c) Cai xin; (d) Fen nie cai; (e) Tai cai.

Seeds of all morphotypes were allowed to germinate on moist filter paper in Petri dishes at $25^{\circ} \mathrm{C}$ until each root was approximately $1.5 \mathrm{~cm}$ long. To increase the number of cells at metaphase, seedlings were treated with $2.0 \mu \mathrm{M}$ 8-oxyquinoline at room temperature for 1.5-2.0 h. After washing three-times for $5 \mathrm{~min}$ each in distilled water, the seedlings were fixed in a fresh 3:1 (v/v) mixture of 100\% ethanol:glacial acetic acid for $24 \mathrm{~h}$, and preserved in $70 \%(\mathrm{v} / \mathrm{v})$ ethanol. Root tips were digested with $4 \%(\mathrm{w} / \mathrm{v})$ cellulase plus $2 \%(\mathrm{w} / \mathrm{v})$ pectinase for approximately $30 \mathrm{~min}$ at $37^{\circ} \mathrm{C}$, after which they were squashed in a drop of $45 \%(\mathrm{v} / \mathrm{v})$ acetic acid. After removing the cover slip by freezing, each slide was air-dried in preparation for FISH.

\section{Fluorescence In situ Hybridization (FISH)}

Table 1 shows all of the synthetic oligonucleotides of 1020 bp that were used as SSR probes. For mono-nucleotides, the rate of $\mathrm{A}$ or $\mathrm{C}$ repeats was representative of itself and $\mathrm{T}$ and G. The AC/AG motifs represent both themselves and the complementary sequences TG/TC. Two di-nucleotides (AT and GC) were not be used in FISH for their selfcomplementary structure (Cuadrado et al., 2008). Hybridization with tri-nucleotides (Jurka and Pethiyagoda, 1995), together with mono- and di-nucleotides, were performed on metaphase chromosomes of five representative cultivars of $B$. rapa ssp. chinensis morphotypes. The SSR sequences were synthesized by Life Technologies (Nanjing, China), and were labeled with digoxigenin-11-dUTP by random primer labeling followed the manufacturer's instructions (Roche). The reaction was performed with $2.0 \mu \mathrm{l}$ of SSR in a $20 \mu \mathrm{l}$ standard reaction by PCR for $3 \mathrm{~h}$ at $37^{\circ} \mathrm{C}$, and the reaction was stopped at $65^{\circ} \mathrm{C}$ for $5 \mathrm{~min}$. Probes were stored at $-20^{\circ} \mathrm{C}$ prior to use in hybridizations.

Fluorescence in situ hybridization was performed as described by Zheng et al. (2014). The post-hybridization slide washing procedure was that of Heslop-Harrison

TABLE 1 | The simple sequence repeat (SSR) probes used in this study.

\begin{tabular}{ll}
\hline Nucleotide repeat type & SSR probes \\
\hline Mono-nucleotides & $(\mathrm{A})_{10},(\mathrm{C})_{10}$ \\
Di-nucleotides & $(\mathrm{AC})_{8},(\mathrm{AG})_{12}$ \\
Tri-nucleotides & $(\mathrm{GCC})_{5},(\mathrm{ACG})_{5},(\mathrm{ACT})_{5},(\mathrm{CAG})_{5},(\mathrm{CAT})_{5}$ \\
& $(\mathrm{CAC})_{5},(\mathrm{ATT})_{5},(\mathrm{AAC})_{5},(\mathrm{AAG})_{5},(\mathrm{AGG})_{5}$ \\
& $(\mathrm{AGC})_{5},(\mathrm{ATC})_{5}$
\end{tabular}

(1991). Detection of digoxigenin was performed by incubating the slides in anti-digoxigenin-rhodamine (Roche) at $37^{\circ} \mathrm{C}$ for $1 \mathrm{~h}$. The chromosomes were then counterstained with $2 \mu \mathrm{g} / \mu \mathrm{l}$ DAPI (Sigma). Re-probing was performed following the method of Cuadrado and Jouve (1994).

\section{Image Acquisition and Analysis}

Fluorescence in situ hybridization signals and images of stained chromosomes were captured using a chilled chargecoupled device (CCD) camera (Axiocam HR, Carl Zeiss, Germany), and images were pseudo-colored and processed using Axiovision software (Carl Zeiss). Detection signals and imaging acquisition were obtained by Zeiss Axio Imager A1 fluorescence microscope. For each SSR motif experiment, we analyzed at least 10 cells with distinct signals. The images from FITC and DAPI staining procedures were recorded separately using a cooled CCD camera. The exposure times depended on the intensity of the signals from each probe. The final images were prepared with Adobe Photoshop, version CS4.

\section{RESULTS}

We used 16 synthetic SSRs as probes for single- and doubletarget FISH. Differences were observed in the abundance and localization of motifs between the different $B$. rapa ssp. chinensis morphotypes, although a general distribution pattern emerged. In all five genomes, most motifs showed a higher density of signal at the centromeric or peri-centromeric regions.

\section{Distribution of Mono- and Di-nucleotide SSRs in the Genomes of B. rapa ssp. chinensis Morphotypes}

We did not detect visible signals for the mono-nucleotide repeats $\mathrm{A}$ and $\mathrm{C}$ on chromosomes of any target genome of $\mathrm{B}$. rapa ssp. chinensis. The di-nucleotide probes, AC and AG, gave visible signals with different patterns among the five samples (Figure 2). Cai xin was the only morphotype in which we detected distinct signals on chromosomes from the two di-nucleotide probes. AG microsatellites showed weak hybridization signals with dispersed patterns in the genomes of Pak-choi and Fen nie cai (Figures 2f,g). 


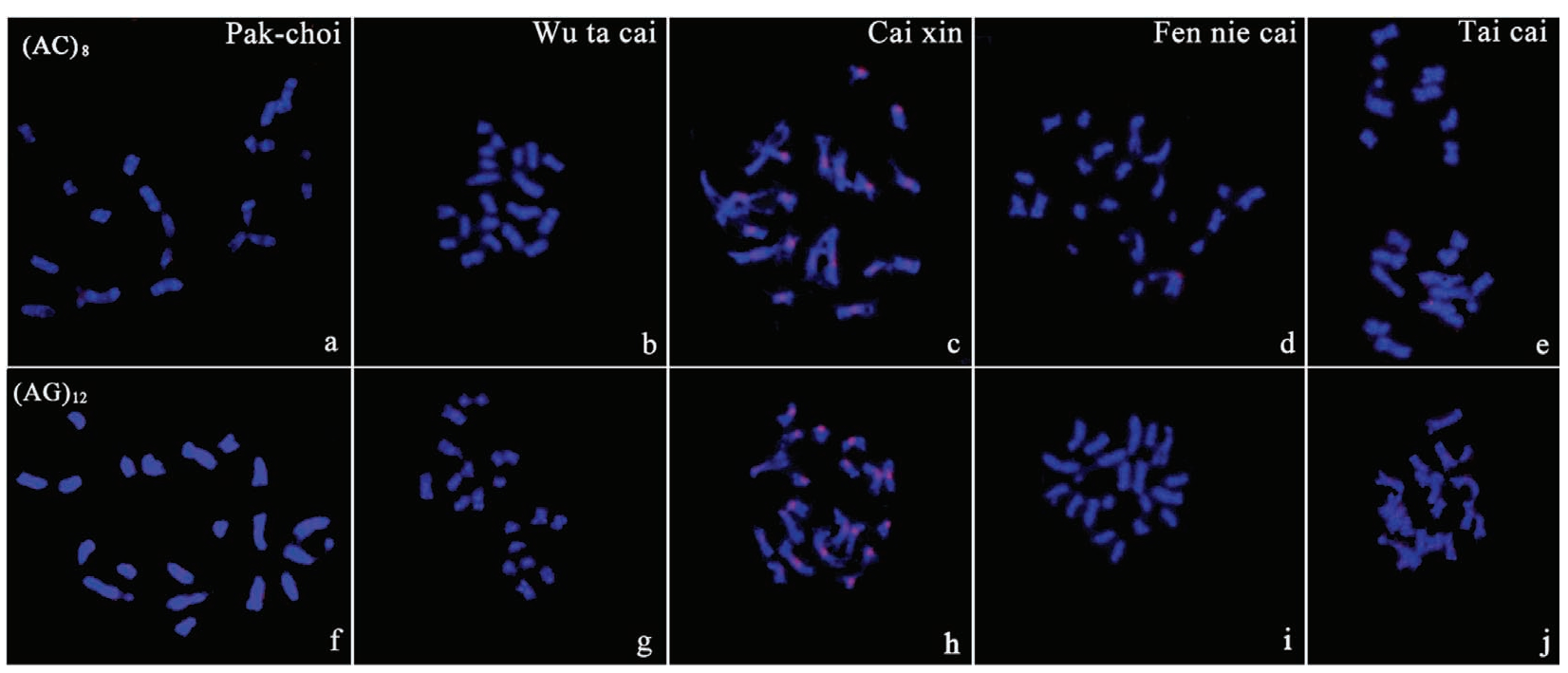

FIGURE 2 | Characterization of the di-nucleotide repeats AC and AG on chromosomes of $B$. rapa ssp. chinensis morphotypes by fluorescent in situ hybridization (FISH) using digoxigenin-labeled probes (detected with red rhodamine). (a-e) (AC) 8 in Pak-choi, Wu ta cai, Cai xin, Fen nie cai, Tai cai; (f-j) $(A G)_{12}$ in Pak-choi, Wu ta cai, Cai xin, Fen nie cai, Tai cai. Chromosomes were counterstained with DAPI.

\section{Physical Characterization of Tri-nucleotide Repeats in B. rapa ssp. chinensis Morphotypes}

Chromosomal Localization Tri-nucleotide Repeats in Pak-choi

Metaphase chromosomes of five B. rapa ssp. chinensis morphotypes were hybridized by re-probing preparations with tri-nucleotide repeat probes (Supplementary Figures S1-S3). All microsatellite motif probes gave in situ hybridization signals on metaphase chromosomes of Pak-choi. Well-defined hybridization signals were produced by ATT, CAC, CAT, AGG, AGC, and ATC probes, and these sequences showed characteristic, motif-dependent distribution patterns (Figure 3). The ATT and CAC probes revealed weak hybridization signals restricted to the centromeres of chromosomes A4 and A5, and the CAT repeat probe gave no signal (Figures 3a-c). In addition, no signal was detected on chromosome A6 after hybridization with CAC, CAT, and ATT repeat probes. The AGC microsatellites co-localized with ATC repeats on Pak-choi metaphase chromosomes with similar intensity, but showed less intensity than AGG repeats (Figures 3d-f). AGG, AGC, and ATC probes showed obvious differences in signal intensity on chromosome A2, AGG being the most intense. These three SSR clusters differed extensively on chromosome A9. AGC and ATC repeats were confined to centromeric regions of this chromosome (Figures 3e,f); however, AGG repeats comprised nearly the entire length of the short arm of A9 (Figure 3d).

\section{Chromosomal Localization Tri-nucleotide Repeats in Wu ta cai}

Differences in the presence/absence and intensity of the hybridization signals were observed on chromosomes of $\mathrm{Wu}$ ta cai after hybridization with tri-nucleotide repeat probes
(Figures 4a,b). All tri-nucleotide repeats were near the centromere on some chromosomes. Polymorphic intercalary signals were observed in two regions of chromosome A2 after hybridizing with AGC and ATC clusters (Figures 4i,k). Hybridization signals of differing intensity were obtained, depending on the SSR motif probe. ATC and AGC, which were present on all chromosomes, showed obvious differences in intensity, the former being more intense. A weak signal on the long arm of chromosome A5, after hybridization with and AGC repeat probe, was observed only after increasing the exposure time of the CCD (compare Figures $4 \mathbf{h}$ and $\mathbf{4 j}$ ).

\section{Chromosomal Localization Tri-nucleotide Repeats in Cai xin}

Variation in intensity and location of the in situ signals were observed on metaphase chromosomes of Cai xin. The signals were confined to the centromere for all tri-nucleotide repeats with differing intensities. Polymorphism in terms of presence/absence was observed in the nucleolus organizing region (NOR). NOR signals were generated after hybridization with AG, CAC, ACT, GCC, and AGG repeats (Figures $\mathbf{5 b}, \mathbf{c}, \mathbf{e}-\mathbf{g}$ ). Visible centromeric signals were observed on chromosome A5 for AG, ACG, CAC, and ACT repeat probes (Figures 5b-e), but not for AC, GCC, and AGG repeats (Figures $\mathbf{5 a}, \mathbf{f}, \mathbf{g}$ ).

\section{Chromosomal Localization Tri-nucleotide Repeats in Fen nie cai}

No specific clusters of SSR-specific signals were observed for tri-nucleotide repeats on chromosomes of Fen nie cai. Polymorphism in terms of presence/absence and intensity of the hybridization signals were observed for some SSRs. The absence signal was revealed from ACT and CAG clusters (Supplementary Figures S1n,s). However, signals of varying intensity were 

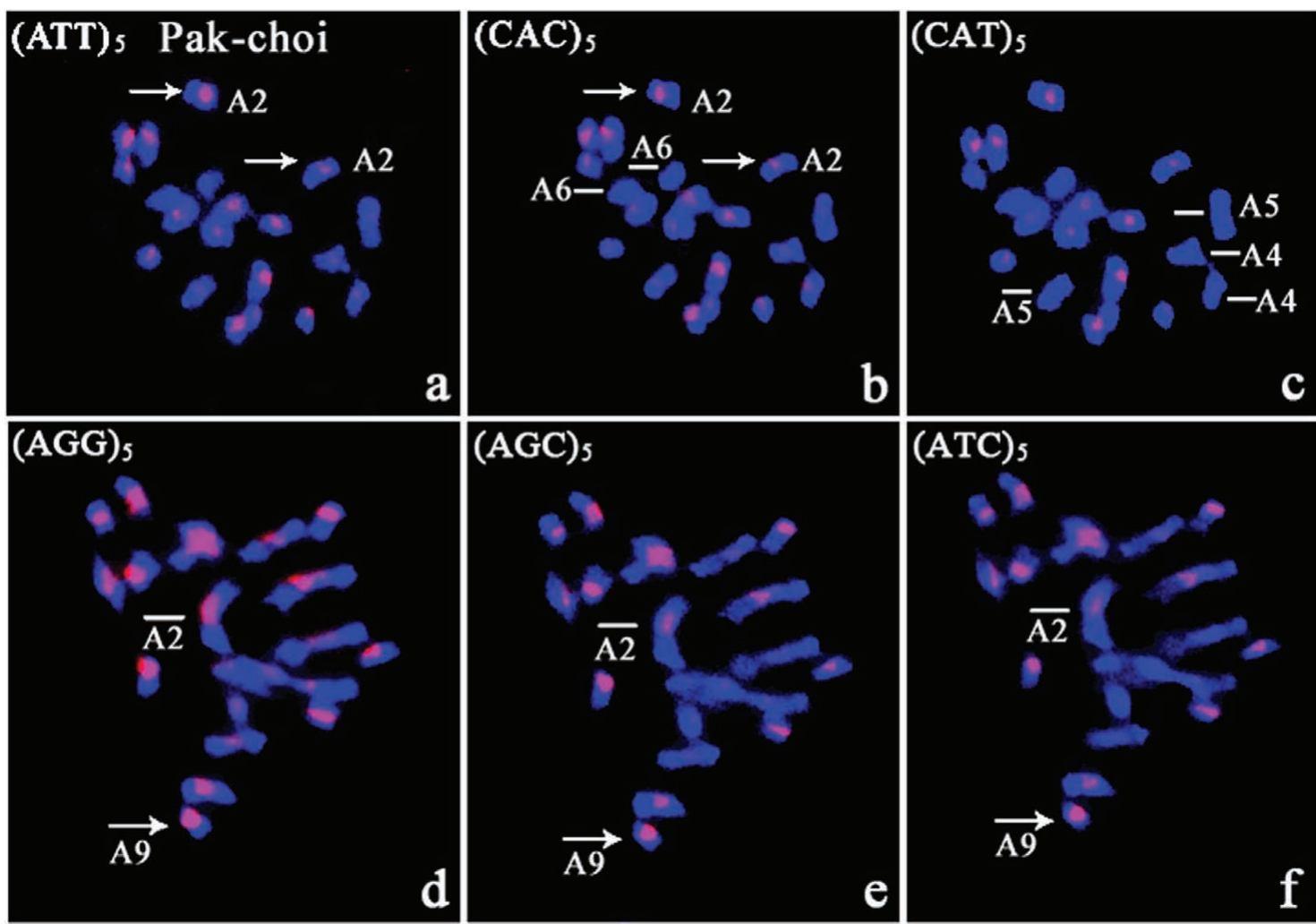

FIGURE 3 | FISH with CAC and ATT repeat probes on metaphase chromosomes of Pak-choi following in situ hybridization with digoxigenin-labeled probes (detected with red rhodamine) and DAPI counterstaining. (a-c) $(A T T)_{5},(C A C)_{5}$, and (CAT) 5 in metaphase chromosomes of Pak choi; arrows indicate the different fluorescent sites from $(\mathrm{ATT})_{5}$ and $(\mathrm{CAC})_{5}$, and lines indicate no signal on chromosome $\mathrm{A} 6$ from $(\mathrm{CAC})_{5}$, chromosomes $\mathrm{A} 4$ and $\mathrm{A} 5$ from $(\mathrm{CAT})_{5}$; (d-f) $(\mathrm{AGG})_{5},(\mathrm{AGC})_{5}$, and $(\mathrm{ATC})_{5}$ in metaphase chromosomes of Pak choi; arrows indicate the different fluorescent signal sites, and lines indicate different intensity fluorescent signals for the SSR loci.

observed on the homologous chromosomes after hybridization with AGG, AGC, and ATC repeat probes (Figures 4c-e).

\section{Chromosomal Localization Tri-nucleotide Repeats in Tai cai}

The most intense and rich patterns of in situ hybridization signals were produced by ACG, ACT, and ATT repeat probes on chromosomes of Tai cai, and were confined to the pericentromeric regions. No specific clustered sites were observed for GCC and AAC repeats (Supplementary Figures S1e and S3t). AAT and CAC repeats were confined around centromeric regions. The ATT repeat probes gave more dispersed and intense centromeric signals, clustered in centromeric and pericentromeric regions, than did the CAC repeats (Figures $\mathbf{4 f , g}$ ). Variations in the number of signal sites were observed on chromosomes A2 and A6. An intercalary signal was observed on the long arms of chromosome A2 (compare Figures 41 with 4n) and A6 (compare Figures $\mathbf{4 m}$ with 4o) from hybridization with ATT repeats, in contrast to only one centromeric signal from CAC repeats (Figures $4 \mathbf{n}, \mathbf{o}$ ). No signals were observed from hybridization with a CAC repeat probe on chromosomes A4, A5, and the NOR, but the ATT repeat probe gave weak signals (Figures 4f,g).

\section{DISCUSSION}

Rogan et al. (2001) showed that base composition, probe length, and chromosomal location contribute to hybridization signal intensity in FISH. In the present study, all probes ranged from 15 to $20 \mathrm{bp}$ in length. The hybridization patterns obtained depended on the base composition of the probes used and chromosomal location. The synthetic SSR probes were labeled by the random primer method, compared with end labeling, which improved the resolution of SSR loci in FISH (Bouilly et al., 2008). Different SSR probes of the same lengths gave different signal intensities, indirectly reflecting the influence of the target size and the copy numbers of the repeat sequences. The SSR probes gave specific hybridization patterns that provide cytogenetic landmarks for chromosome identification.

\section{Different SSR Distribution Patterns on Chromosomes Depend on the Species Analyzed}

Sonah et al. (2011) showed that mono-, di-, and tri-nucleotide repeats compose the major proportion of SSRs in plant genomes. For tri-nucleotides, A/T-rich repeats (e.g., AAC/GTT, AAG/CTT, and AAT/ATT) were predominant in dicot species. In the 

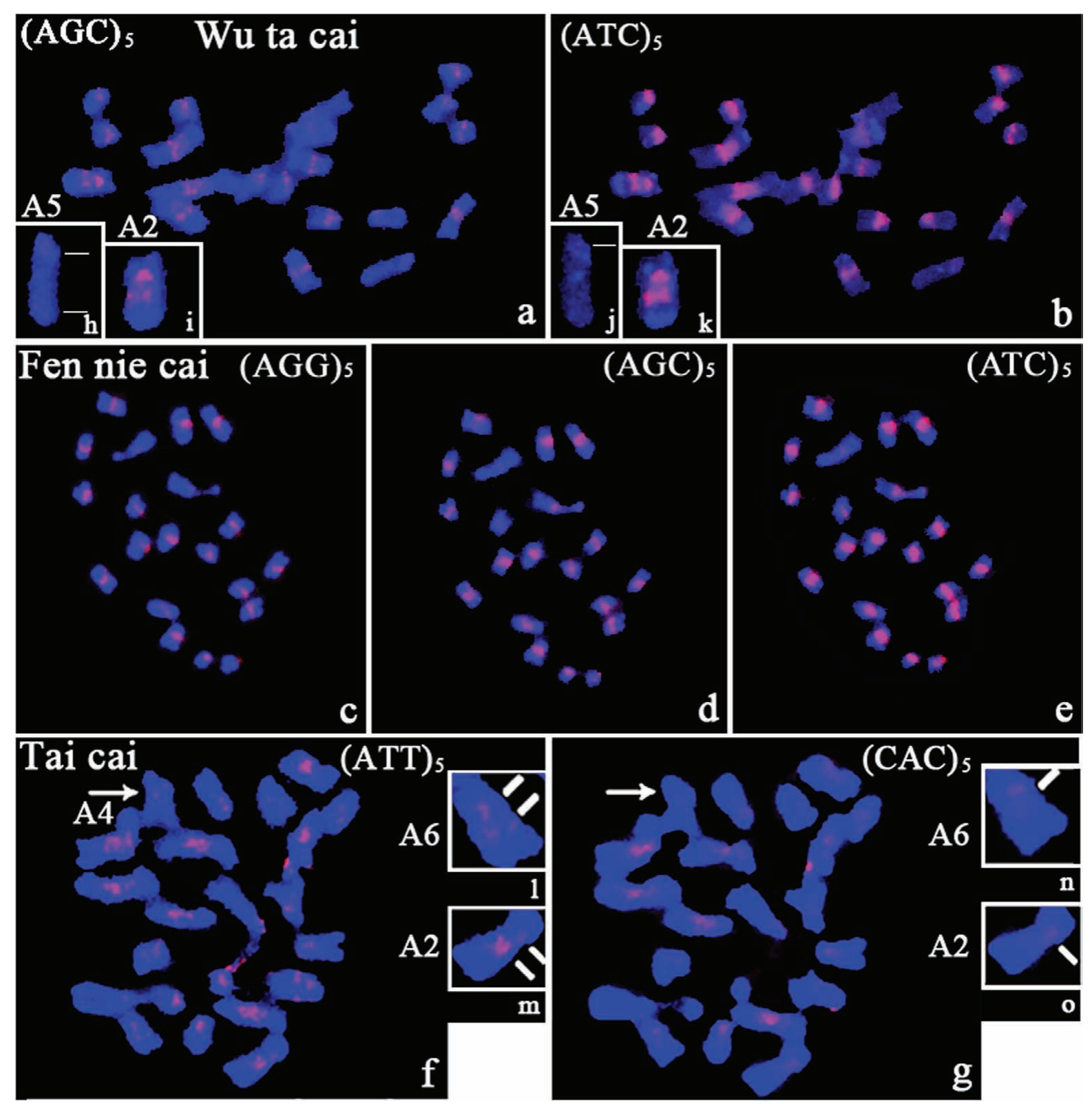

FIGURE 4 | Photomicrographs showing the distribution of several tri-nucleotide SSRs (AGG, AGC, and ATC) on metaphase chromosomes of Wu ta

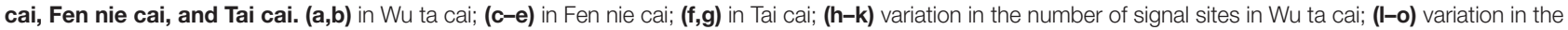
number of signal sites in Tai cai; lines indicate the number of fluorescent signals in Wu ta cai and Tai cai; arrows indicate different intensity fluorescent signals. Chromosomes were counterstained with DAPI.

monocot barley, however, repeated AAT SSR motifs gave poor hybridization signals (Cuadrado and Jouve, 2007b). Morgante et al. (2002) found that GCC repeats accounted for half of the tri-nucleotide repeats in rice, whereas they were rare in dicots. Lawson and Zhang (2006) examined the most common mono(A/T) and di-nucleotide (AT and AG) repeats in the Arabidopsis genome, and found that polyA/T repeats were predominant, while polyC/G repeats were rare (Sonah et al., 2011). The distribution of $\mathrm{AC}$ and $\mathrm{AG}$ repeats were linked to the euchromatic and heterochromatic genomic regions, respectively (Cuadrado and Jouve, 2007a). The di-nucleotide AG repeats were located on all chromosomes in Dendrobium aphyllum and D. aggregatum (Begum et al., 2009), but were exclusively concentrated at the centromeres in Triticum (Carmona et al., 2013). The most abundant repeat motifs were A (28.8\%), AG (15.4\%), AT (13.7\%), and AAG (13.3\%) clusters, reflecting the $\mathrm{A} / \mathrm{T}$ rich nature of the $B$. rapa genome (Hong et al., 2007). In this study, nearly all the di- and tri-nucleotide SSRs were detected on metaphase chromosomes of Cai xin and Pak-choi, inferring that these two morphotypes have more types and increased abundance of SSRs 

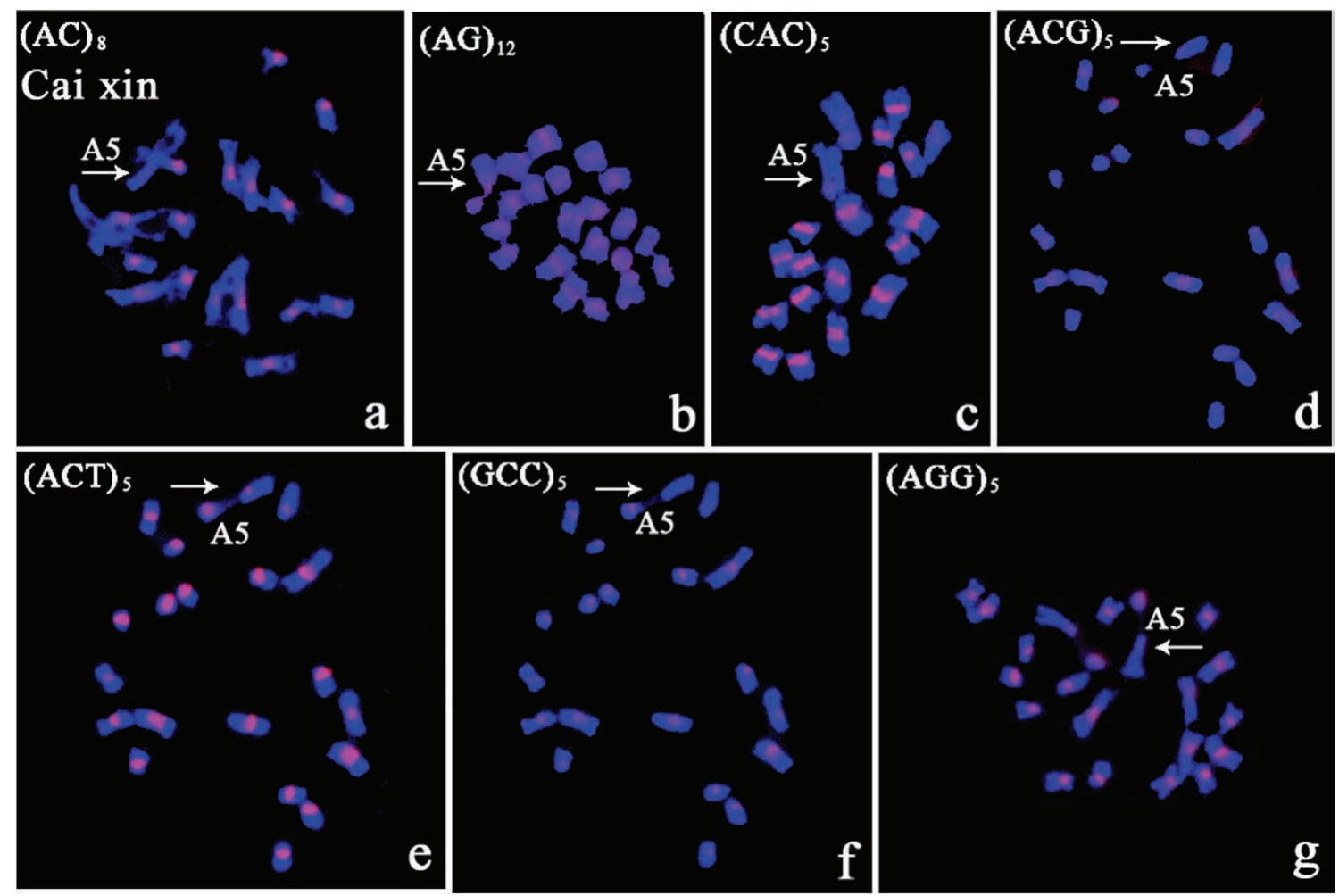

FIGURE 5 | The polymorphic characterization of the chromosome bearing the NOR in Cai xin following in situ hybridization with digoxigenin-labeled probes (detected with red rhodamine) and DAPI counterstaining. (a) (AC) $)_{8} ;$ (b) $(\mathrm{AG})_{12} ;$ (c) $(\mathrm{CAC})_{5} ;$ (d) $(\mathrm{ACG})_{5} ;$ (e) (ACT) $)_{5} ;$ (f) $(\mathrm{GCC})_{5} ;$ (g) $(\mathrm{AGG})_{5} ;$ arrows indicate chromosome A5; no signal was detected on the NOR in (a,d); fluorescent signal on the NOR in (c); weak centromeric signal in (d); signals on the NOR and the centromere in $\mathbf{( b , e , g )}$.

compared to the other three morphotypes (Wu ta cai, Fen nie cai, and Tai cai).

Various types of SSR motifs display taxon-specific patterns in the genomes of prokaryotes and eukaryotes (Toth et al., 2000). For example, the most intense hybridization signals were produced by the AGG, AAG, and AAC tri-nucleotide probes in barley (Cuadrado and Jouve, 2007b). The AAC clusters showed the same distribution patterns as AAG repeats in wheat (Cuadrado and Schwarzacher, 1998). AAG repeat units are major contributors to the genomes of dicots (Sonah et al., 2011); they are preferentially associated with peri-centromeric heterochromatin in Hordeum species (Carmona et al., 2013), and generally reflect the distribution of heterochromatin and the C-banding pattern in wheat (Cuadrado and Jouve, 1994). AAC repeats are organized in a more dispersed manner, with centromeric regions being largely excluded in chickpea and tomato (Gortner et al., 1998; Gindullis et al., 2001). Some repeats, such as CAG, CAC, and ACG, have specific hybridization sites restricted to the centromeres of metaphase chromosomes in barley (Cuadrado and Jouve, 2007b).

Despite the stable chromosome number and similarities in chromosome size and morphology, differences in numbers and distribution of SSR blocks have been observed among B. rapa ssp. chinensis morphotypes. The ATT repeat probe gave two signals with different intensities on chromosomes A2 and A6 of Tai cai, indicating that this SSR experienced chromosome-specific accumulation during evolution, as did AGG and ATC repeats in $\mathrm{Wu}$ ta cai. In addition, several SSRs (ATT, CAC, AGG, AGC, and ATC) also showed chromosome-specific signals on marker chromosomes of Pak-choi. These three morphotypes may have resulted from chromosomal recombination during speciation and development.

\section{The Different Distribution Patterns on Chromosomes Depend on the SSR Probes Used}

A negative correlation has been observed between repeat numbers and the total length of repeat units in both monocots and dicots (Cavagnaro et al., 2010; Gao et al., 2011; Sonah et al., 2011). Mono-nucleotide repeats of $\mathrm{A}$ are the most abundant repeats among all SSRs analyzed (Gao et al., 2011); however, no visible signal was generated, partly implying their dispersed distribution along chromosomes, or may be related to the centromeric function (Cuadrado and Jouve, 2007a). In this work, the di-nucleotide repeats AC and AG only showed distinct in situ hybridization signals on chromosomes of Cai xin, although there were weak signals in Pak-choi and Fen nie cai for $A G$ repeats. In addition, three tri-nucleotide repeat probes (AAG, AGG, and AGC) gave distinct signals on all analyzed genomes. Di-nucleotide AG and tri-nucleotide AAG repeats are 
relatively abundant in the B. rapa genome (Hong et al., 2007). AG-rich microsatellites might be the most prevalent SSRs that cluster around centromeric regions in B. rapa ssp. chinensis morphotypes.

We found that some tri-nucleotide repeats distinctly colocalized within the same genome, although the signals differed in intensity, which could be explained by their intermixed structure at the given loci. Some SSR probes gave weak or dispersed signals at the same physical position, which could possibly be due to closely linked blocks of repeated sequences. Here, we show that individual SSRs vary widely in their relative proportions at the chromosome level, and that the distribution of SSRs along the chromosomes is non-random. The characteristic patterns of SSR distribution show that centromeric regions are more densely populated than the central regions (Rogan et al., 2001). The similar distribution patterns of some SSR motifs indicate that long stretches of different SSRs are of functional importance, and could possibly represent an ancient component of plant genomes (Cuadrado et al., 2000; Cuadrado and Jouve, 2007b). These results are supported by evidence showing that microsatellites display relatively uniform coverage in the genome, and that there are taxon-specific distribution patterns among B. rapa ssp. chinensis morphotypes. Our results suggest that SSR repeats are the major component of the satellite DNA fraction, and show evolutionary conservation among $B$. rapa ssp. chinensis morphotypes.

\section{Relationship between SSRs and Centromeres}

In most higher eukaryotic organisms, chromosomal centromeres are composed of long arrays of satellite repeat sequences and retro-transposons (Henikoff et al., 2001; Jiang et al., 2003). The centromeric and distal regions play important roles during mitosis and meiosis (Li et al., 2002; Hong et al., 2006), and highly divergent sequences are present in the peri-centromeric regions (Wang et al., 2012). The repetitive DNA sequences frequently form clusters within heterochromatin blocks, which are predominantly concentrated at peri-centromeric regions and have been detected in plants with small and compact genomes (Cuadrado and Jouve, 2010; Falistocco and Marconi, 2013). In the present work, most in situ SSR signals were confined to centromeric or adjacent regions on B. rapa ssp. chinensis chromosomes. This distributional characterization may be related to their effect on DNA replication, chromatin organization, and the cell cycle.

\section{Evolutionary Trends of SSRs among B. rapa ssp. chinensis Genomes}

The relationship between microsatellites and chromosomal evolution has not been clearly documented. The frequency of repeats decreases exponentially with their length, type, and number of SSR motif repeats. This characterization appears to be more conservative in coding than non-coding sequences (Cavagnaro et al., 2010; Sonah et al., 2011; Shi et al., 2013), and less pronounced for di-nucleotides compared to longer repeat types (Cavagnaro et al., 2010). The trends for various repeat types are similar between different chromosomes within the same genome, but the density of repeats may vary between different chromosomes in the same species (Schafer et al., 1986; Katti et al., 2001). Sonah et al. (2011) found species-specific accumulation of particular motif repeats. Variation is present between related species in terms of the abundance and chromosomal distribution of SSR clusters among morphotypes. Schmidt and HeslopHarrison (1996) demonstrated that microsatellites, representing a substantial fraction of the genome, showed chromosomespecific amplification in plants. High levels of polymorphism and heterozygosity between homologs, in terms of the distribution of AAG and AAC repeats, was shown for out-breeding species in the Secale strictum species complex (Cuadrado and Jouve, 2002).

Our results suggest that SSR sequences are more predisposed to being amplified or deleted as a result of independent events. The balance among SSR sequences generated by strandslippage replication, or recombination and repair mechanisms, cannot be the only explanation for the observed differences in their chromosomal distributions. Another possible explanation would involve selection pressure or mutation (Li et al., 2002). The different chromosomal positions of SSRs involved in the regulation of gene expression (Lawson and Zhang, 2006; Gao et al., 2011), could indicate their underestimated roles in genome evolution (Cuadrado et al., 2008).

The microsatellite sequences analyzed here showed similar chromosome distribution polymorphism patterns, inferring that these SSR loci may result from convergent evolution. However, they differed in intensity or position, indicating that microsatellite repeats can contract or expand over a very short evolutionary time frame (Iwata et al., 2013). The wide distribution of SSRs, and the fact that their positions are restricted to chromosomal centromeres, as revealed by FISH, suggested a general model for the parallel chromosome evolution of repeatrich heterochromatin in B. rapa ssp. chinensis.

Carmona et al. (2013) suggested that changes in the amount and distribution of tandem repetitive DNA sequences are major driving forces of genome evolution and speciation. The different regions are thought to undergo different selection pressures (Morgante et al., 2002), which might account for different motif preferences and frequencies among chromosomes. The evolutionary dynamics of microsatellites is generally consistent with plant divergence and evolution (Shi et al., 2013), and the distribution of microsatellites is related to the history of genome evolution and selective constraints (Morgante et al., 2002). The variation in SSRs at the chromosome level may be the result of adaptive divergence, or selection resulting from the stress response among species and populations (Cavagnaro et al., 2010; Gao et al., 2011; Carmona et al., 2013). Whether SSRs are under selection or are neutral as has been reported (Ellegren, 2004), and can be used for exploring the dynamics of the evolutionary process (Santos et al., 2010), will require study.

\section{AUTHOR CONTRIBUTIONS}

S-nZ and J-sZ: Designed the experiment. X-lH and GB: Provided the materials. J-sZ and C-zS: Performed the experiment. J-sZ: Analyzed the data and wrote the manuscript. 


\section{FUNDING}

This work was supported by Science and Technology Pillar Program of Jiangsu Province (BE2013429), the Agricultural science and technology independent innovation funds of Jiangsu Province [CX(13)2006], the National Fund of Hebei Province, China (Project No. C2015407058) and Scientific Research Project of Hebei Province China (Project No. QN2016110).

\section{SUPPLEMENTARY MATERIAL}

The Supplementary Material for this article can be found online at: http://journal.frontiersin.org/article/10.3389/fpls.2016.01049

FIGURE S1 | Photomicrographs showing the distribution of the tri-nucleotide repeats $(\mathrm{GCC})_{5},(\mathrm{ACG})_{5},(\mathrm{ACT})_{5},(\mathrm{CAG})_{5}$ on metaphase

\section{REFERENCES}

Beckmann, J. S., and Weber, J. L. (1992). Survey of human and rat microsatellites. Genomics 12, 627-631. doi: 10.1016/0888-7543(92)90285-Z

Begum, R., Alam, S. S., Menzel, G., and Schmidt, T. (2009). Comparative molecular cytogenetics of major repetitive sequence families of three Dendrobium species (Orchidaceae) from Bangladesh. Ann. Bot. 104, 863-872. doi: 10.1093/aob/mcp178

Bouilly, K., Chaves, R., Leitao, A., Benabdelmouna, A., and Guedes-Pinto, H. (2008). Chromosomal organization of simple sequence repeats in the Pacific oyster (Crassostrea gigas): (GGAT)4, (GT)7 and (TA)10 chromosome patterns. J. Genet. 87, 119-125. doi: 10.1007/s12041-008-0018-2

Carmona, A., Friero, E., de Bustos, A., Jouve, N., and Cuadrado, A. (2013). Cytogenetic diversity of SSR motifs within and between Hordeum species carrying the $\mathrm{H}$ genome: $H$. vulgare L. and H. bulbosum L. Theor. Appl. Genet. 126, 949-961. doi: 10.1007/s00122-012-2028-y

Cavagnaro, P. F., Senalik, D. A., Yang, L. M., Simon, P. W., Harkins, T. T., Kodira, C. D., et al. (2010). Genome-wide characterization of simple sequence repeats in cucumber (Cucumis sativus L.). BMC Genomics 11:569. doi: 10.1186/14712164-11-569

Cheng, F., Mandáková, T., Wu, J., Xie, Q., Lysak, M. A., and Wang, X. W. (2013). Deciphering the diploid ancestral genome of the mesohexaploid Brassica rapa. Plant Cell 25, 1541-1554. doi: 10.1105/tpc.113.110486

Cuadrado, A., Cardoso, M., and Jouve, N. (2008). Physical organisation of simple sequence repeats (SSRs) in Triticeae: structural, functional and evolutionary implications. Cytogenet. Genome Res. 120, 210-219. doi: 10.1159/000121069

Cuadrado, A., and Jouve, N. (1994). Mapping and organization of highly-repeated DNA sequences by means of simultaneous and sequential FISH and C-banding in 6x-triticale. Chromosome Res. 2, 331-338. doi: 10.1007/BF01552727

Cuadrado, A., and Jouve, N. (2002). Evolutionary trends of different repetitive DNA sequences during speciation in the genus Secale. J. Hered. 93, 339-345. doi: 10.1093/jhered/93.5.339

Cuadrado, A., and Jouve, N. (2007a). Similarities in the chromosomal distribution of AG and AC repeats within and between Drosophila, human and barley chromosomes. Cytogenet. Genome Res. 119, 91-99. doi: 10.1159/000109624

Cuadrado, A., and Jouve, N. (2007b). The nonrandom distribution of long clusters of all possible classes of tri-nucleotide repeats in barley chromosomes. Chromosome Res. 15, 711-720. doi: 10.1007/s10577-007-1156-8

Cuadrado, A., and Jouve, N. (2010). Chromosomal detection of simple sequence repeats (SSRs) using nondenaturing FISH (ND-FISH). Chromosoma 119, 495503. doi: 10.1007/s00412-010-0273-x

Cuadrado, A., and Jouve, N. (2011). Novel simple sequence repeats (SSRs) detected by ND-FISH in heterochromatin of Drosophila melanogaster. BMC Genomics 12:205. doi: 10.1186/1471-2164-12-205

Cuadrado, A., and Schwarzacher, T. (1998). The chromosomal organization of simple sequence repeats in wheat and rye genomes. Chromosoma 107, 587-594. doi: $10.1007 / \mathrm{s} 004120050345$ chromosomes of five B. rapa ssp. chinensis morphotypes after in situ hybridization with digoxigenin-labeled probes (detected with red rhodamine) and DAPI counterstaining. $(\mathbf{a}, \mathbf{f}, \mathbf{k}, \mathbf{p})$ in Pak-choi; $(\mathbf{b}, \mathbf{g}, \mathbf{l}, \mathbf{q})$ in Wu ta

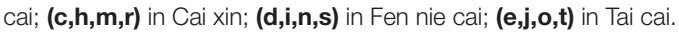

FIGURE S2 | Photomicrographs showing the distribution of (AAG) ${ }_{5}$, $(A G G)_{5},(A G C)_{5}$, and $(A T C)_{5}$ repeats on metaphase chromosomes of five $B$. rapa ssp. chinensis morphotypes after in situ hybridization with digoxigenin-labeled probes (detected with red rhodamine) and DAPI

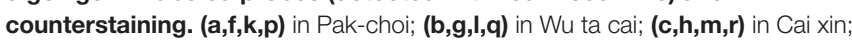
$(\mathbf{d}, \mathbf{i}, \mathbf{n}, \mathbf{s})$ in Fen nie cai; $(\mathbf{e}, \mathbf{j}, \mathbf{o}, \mathbf{t})$ in Tai cai.

FIGURE S3 | Photomicrographs showing the distribution of (CAT) $)_{5},(C A C)_{5}$, $(\mathrm{ATT})_{5}$, and $(\mathrm{AAC})_{5}$ repeats on metaphase chromosomes of five $B$. rapa ssp. chinensis morphotypes after in situ hybridization with digoxigenin-labeled probes (detected with red rhodamine) and DAPI

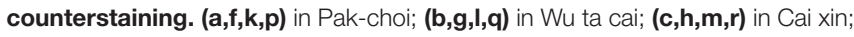
$(\mathbf{d}, \mathbf{i}, \mathbf{n}, \mathbf{s})$ in Fen nie cai; $(\mathbf{e}, \mathbf{j}, \mathbf{o}, \mathbf{t})$ in Tai cai.

Cuadrado, A., Schwarzacher, T., and Jouve, N. (2000). Identification of different chromatin classes in wheat using in situ hybridization with simple sequence repeat oligonucleotides. Theor. Appl. Genet. 101, 711-717. doi: $10.1007 / \mathrm{s} 001220051535$

Ellegren, H. (2004). Microsatellites: simple sequences with complex evolution. Nat. Rev. Genet. 5, 435-445. doi: 10.1038/nrg1348

Falistocco, E., and Marconi, G. (2013). Cytogenetic characterization by in situ hybridization techniques and molecular analysis of 5S rRNA genes of the European hazelnut (Corylus avellana). Genome 56, 155-159. doi: 10.1139/gen2013-0045

Fang, P., Chen, F. B., Yao, Q. L., Yang, K. C., Zhou, G. F., Fan, Y. H., et al. (2013). Analysis of genetic diversity in the tuber mustard (Brassica juncea var. tumida Tsen et Lee) in the Yangtze river basin of China. Genet. Resour. Crop Evol. 60, 129-143. doi: 10.1007/s10722-012-9821-y

Gao, C. H., Tang, Z. L., Yin, J. M., An, Z. S., Fu, D. H., and Li, J. N. (2011). Characterization and comparison of gene-based simple sequence repeats across Brassica species. Mol. Genet. Genomics 286, 161-170. doi: 10.1007/s00438-0110636-X

Gao, L. F., Tang, J. F., Li, H. W., and Jia, J. Z. (2003). Analysis of microsatellites in major crops assessed by computational and experimental approaches. Mol. Breed. 12, 245-261. doi: 10.1023/A:1026346121217

Gindullis, F., Desel, C., Galasso, I., and Schmidt, T. (2001). The large-scale organization of the centromeric region in Beta species. Genome Res. 11, 253265. doi: 10.1101/gr.162301

Gladis, T., and Hammer, K. (1992). The Gatersleben Brassica collection-Brassica juncea, B. napus, B. nigra and B. rapa (Germ., Engl. summary). Feddes Repert. 103, 469-507. doi: 10.1002/fedr.19921030704

Gortner, G., Nenno, M., Weising, K., Zink, D., Nagl, W., and Kahl, G. (1998). Chromosomal localization and distribution of simple sequence repeats and the Arabidopsis-type telomere sequence in the genome of Cicer arietinum L. Chromosome Res. 6, 97-104. doi: 10.1023/A:10092828 28236

Henikoff, S., Ahmad, K., and Malik, H. S. (2001). The centromere paradox: stable inheritance with rapidly evolving DNA. Science 293, 1098-1102. doi: $10.1126 /$ science. 1062939

Heslop-Harrison, J. S. (1991). The molecular cytogenetics of palnts. J. Cell Sci. 100, 15-21.

Hong, C. P., Piao, Z. Y., Kang, T. W., Batley, J., Yang, T. J., Hur, Y. K., et al. (2007). Genomic distribution of simple sequence repeats in Brassica rapa. Mol. Cells 23, 349-356.

Hong, C. P., Plaha, P., Koo, D. H., Yang, T. J., Choi, S. R., Lee, Y. K., et al. (2006). A survey of the Brassica rapa genome by BAC-End sequence analysis and comparison with Arabidopsis thaliana. Mol. Cells 22, 300-307.

Iwata, A., Tek, A. L., Richard, M. M. S., Abernathy, B., Fonseca, A., Schmutz, J., et al. (2013). Identification and characterization of functional centromeres of the common bean. Plant J. 76, 47-60. doi: 10.1111/tpj. 12269 
Jiang, J. M., Birchler, J. A., Parrott, W. A., and Dawe, R. K. (2003). A molecular view of plant centromeres. Trends Plant Sci. 8, 570-575 doi: 10.1016/j.tplants.2003.10.011

Jurka, J., and Pethiyagoda, C. (1995). Simple repetitive DNA-sequeences from primates-compilation and analysis. J. Mol. Evol. 40, 120-126. doi: 10.1007/BF00167107

Katti, M. V., Ranjekar, P. K., and Gupta, V. S. (2001). Differential distribution of simple sequence repeats in eukaryotic genome sequences. Mol. Biol. Evol. 18, 1161-1167. doi: 10.1093/oxfordjournals.molbev.a003903

Koo, D. H., Plaha, P., Lim, Y. P., Hur, Y., and Bang, J. W. (2004). A highresolution karyotype of Brassica rapa ssp. pekinensis revealed by pachytene analysis and multicolor fluorescence in situ hybridization. Theor. Appl. Genet. 109, 1346-1352. doi: 10.1007/s00122-004-1771-0

Lawson, M. J., and Zhang, L. Q. (2006). Distinct patterns of SSR distribution in the Arabidopsis thaliana and rice genomes. Genome Biol. 7:R14. doi: 10.1186/gb2006-7-2-r14

Li, C., Huang, B., Yan, X. H., Wang, L. J., Yang, Q., and Wei, W. H. (2010). Cloning, identification and characterization of a repetitive sequence flanking telomere and homologous to canrep in Brassica napus. Bot. Stud. 51, 421-430.

Li, Y., Korol, A. B., Fahima, T., Beiles, A., and Nevo, E. (2004). Microsatellites within genes: structure, function and evolution. Mol. Biol. Evol. 21, 991-1007 doi: 10.1093/molbev/msh073

Li, Y. C., Korol, A. B., Fahima, T., Beiles, A., and Nevo, E. (2002). Microsatellites: genomic distribution, putative functions and mutational mechanisms: a review. Mol. Ecol. 11, 2453-2465. doi: 10.1046/j.1365-294X.2002.01643.x

Lohe, A. R., Hilliker, A. J., and Roberts, P. A. (1993). Mapping simple repeated DNA sequences in heterochromatin of Drosophila melanogaster. Genetics 134, 1149-1174. doi: 10.1016/0168-9525(93)90135-5

Lysak, M. A., Koch, M. A., Pecinka, A., and Schubert, I. (2005). Chromosome triplication found across the tribe Brassiceae. Genome Res. 15, 516-525. doi: 10.1101/gr.3531105

Morgante, M., Hanafey, M., and Powell, W. (2002). Microsatellites are preferentially associated with nonrepetitive DNA in plant genomes. Nat. Genet. 30, 194-200. doi: 10.1038/ng822

Mortimer, J. C., Batley, J., Love, C. G., Logan, E., and Edwards, D. (2005). Simple sequence repeat (SSR) and GC distribution in the Arabidopsis thaliana genome. J. Plant Biotechnol. 7, 17-25.

Nagaharu, U. (1935). Genome analysis in Brassica with special reference to the experimental formation of $B$. napus and peculiarmode of fertilization. Jpn. J. Bot. 7, 389-452.

Nagaki, K., Cheng, Z. K., Ouyang, S., Talbert, P. B., Kim, M., Jones, K. M., et al. (2004). Sequencing of a rice centromere uncovers active genes. Nat. Genet. 36, 138-145. doi: 10.1038/ng1289

Nanda, I., Zischler, H., Epplen, C., Guttenbach, M., and Schmid, M. (1991). Chromosomal organization of simple repeated DNA-sequeences used for DNA fingerprinting. Electrophoresis 12, 193-203. doi: 10.1002/elps.1150120216

Pedersen, C., Rasmussen, S. K., and Linde-Laursen, I. (1996). Genome and chromosome identification in cultivated barley and related species of the Triticeae (Poaceae) by in situ hybridization with the GAA-satellite sequence. Genome 39, 93-104. doi: 10.1139/g96-013

Rogan, P. K., Cazcarro, P. M., and Knoll, J. H. M. (2001). Sequence-based design of single-copy genomic DNA probes for fluorescence in situ hybridization. Genome Res. 11, 1086-1094. doi: 10.1101/gr.171701

Santos, J., Serra, L., Sole, E., and Pascual, M. (2010). FISH mapping of microsatellite loci from Drosophila subobscura and its comparison to related species. Chromosome Res. 18, 213-226. doi: 10.1007/s10577-010-9112-4

Schafer, R., Ali, S., and Epplen, J. T. (1986). The organization of the evolutionarily conserved GATA GACA repeats in the mouse genome. Chromosoma 93, 502510. doi: 10.1007/BF00386791

Schmidt, T., and Heslop-Harrison, J. S. (1996). The physical and genomic organization of microsatellites in sugar beet. Proc. Natl. Acad. Sci. U.S.A. 93, 8761-8765. doi: 10.1073/pnas.93.16.8761
Shi, J. Q., Huang, S. M., Fu, D. H., Yu, J. Y., Wang, X. F., Hua, W., et al. (2013). Evolutionary dynamics of microsatellite distribution in plants: insight from the comparison of sequenced Brassica, Arabidopsis and other angiosperm species. PLoS ONE 8:e59988. doi: 10.1371/journal.pone.0059988

Snowdon, R. J. (2007). Cytogenetics and genome analysis in Brassica crops. Chromosome Res. 15, 85-95. doi: 10.1007/s10577-006-1105-y

Sonah, H., Deshmukh, R. K., Sharma, A., Singh, V. P., Gupta, D. K., Gacche, R. N., et al. (2011). Genome-wide distribution and organization of microsatellites in plants: an insight into marker development in Brachypodium. PLoS ONE 6:e21298. doi: 10.1371/journal.pone.0021298

Tautz, D., and Renz, M. (1984). Simple sequences are ubiquitous repetitive components of eukaryotic genomes. Nucleic Acids Res. 12, 4127-4138. doi: $10.1093 /$ nar/12.10.4127

Toth, G., Gaspari, Z., and Jurka, J. (2000). Microsatellites in different eukaryotic genomes: survey and analysis. Genome Res. 10, 967-981. doi: 10.1101/gr.10.7.967

Viehoever, A. (1920). Studies in mustard seeds and substitutes: I. Chinese colza (Brassica campestris chinoleifera Viehoever). J. Agric. Res. 20, 117-140.

Wang, K., Zhang, W. P., Gao, Y. J., Zhang, Z. X., Zheng, D. W., Zhou, B. L., et al. (2012). Localization of high level of sequence conservation and divergence regions in cotton. Theor. Appl. Genet. 124, 1173-1182. doi: 10.1007/s00122-0111777-3

Wang, X., Wang, H., Wang, J., Sun, R., Wu, J., Liu, S., et al. (2011). The genome of the mesopolyploid crop species Brassica rapa. Nat. Genet. 43, 1035-1039. doi: 10.1038/ng.919

Yang, T. J., Lee, S., Chang, S. B., Yu, Y., Jong, H., and Wing, R. A. (2005). Indepth sequence analysis of the tomato chromosome 12 centromeric region: identification of a large CAA block and characterization of pericentromere retrotranposons. Chromosoma 114, 103-117. doi: 10.1007/s00412-005-0342-8

Yu, S. C., Zhang, F. L., Wang, X. Y., Zhao, X. Y., Zhang, D. S., Yu, Y. J., et al. (2010). Genetic diversity and marker-trait associations in a collection of Pakchoi (Brassica rapa L. ssp Chinensis Makino) accessions. Genes Genomics 32, 419-428. doi: 10.1007/s13258-010-0033-6

Zhebentyayeva, T. N., Reighard, G. L., Gorina, V. M., and Abbott, A. G. (2003). Simple sequence repeat (SSR) analysis for assessment of genetic variability in apricot germplasm. Theor. Appl. Genet. 106, 435-444.

Zheng, J. S., Sun, C. Z., Xiao D., Zhang, S. N., Bonnema G., and Hou, X. L. (2015). Karyotype variation and conservation in morphotypes of non-heading Chinese cabbage. Plant Syst. Evol. 301, 1781-1791. doi: 10.1007/s00606-0141177-7

Zheng, J. S., Sun, C. Z., Zhang, S. N., and Hou, X. L. (2014). Karyotype of mitotic metaphase and meiotic diakinesis in non-heading Chinese cabbage. Plant Syst. Evol. 300, 295-302. doi: 10.1007/s00606-013-0882-y

Zhu, X. C., Raman, H., Wu, H. W., Lemerle, D., Burrows, G. E., and Stanton, R. (2013). Development of SSR markers for genetic analysis of rilverleaf nightshade (Solanum elaeagnifolium) and related rpecies. Plant Mol. Biol. Rep. 31, 248-254. doi: 10.1007/s11105-012-0473-z

Conflict of Interest Statement: The authors declare that the research was conducted in the absence of any commercial or financial relationships that could be construed as a potential conflict of interest.

The reviewer ST and handling Editor declared their shared affiliation, and the handling Editor states that the process nevertheless met the standards of a fair and objective review.

Copyright (c) 2016 Zheng, Sun, Zhang, Hou and Bonnema. This is an open-access article distributed under the terms of the Creative Commons Attribution License (CC BY). The use, distribution or reproduction in other forums is permitted, provided the original author(s) or licensor are credited and that the original publication in this journal is cited, in accordance with accepted academic practice. No use, distribution or reproduction is permitted which does not comply with these terms. 